\title{
Experiencias paramétricas en la formación del arquitecto: ensayo de aplicación a los talleres iniciales
}

Parametric experiences in architect's education: case of application in a basic design studio

> Miriam Bessone

Universidad Nacional del Litoral, Argentina. mbessoneyarqcolaborativas@yahoo.com.ar

> Matias Dalla Costa

Universidad Nacional del Litoral, Argentina. matiasdallacosta@hotmail.com
> María Elena Tosello

Universidad Nacional del Litoral, Argentina. mtosello@fadu.unl.edu.ar

\begin{abstract}
In the actual textual landscape, images work activating cognitive and behavioral processes, disrupting the anthropological context of critical thinking based on logical-formal reasoning. Different types of languages -carrying specific signs and syntax-entwine to oral and written text, augmenting the differences and introducing new problematic in education, situation that requires to be investigate, debate and adequate. From this statement and through the presentation of a didactic experience of parametric systems application in a beginning design studio of Architecture, this paper aims to contribute to the discussion of which are the notions and basic strategies needed to initiate significant learning process that allow to think and design starting from emergent design languages.
\end{abstract}

Keywords: Didactic Strategies; Design Strategies; Parametric Design; Architecture

\section{Introducción}

En el paisaje cultural contemporáneo comienza a predominar un sujeto joven que ha producido un desplazamiento en los modos de acceder al conocimiento, operar y relacionarse socialmente. Videojuegos, pantallas táctiles, sensores, etc., son dispositivos que expanden su experiencia sensorial y se entrelazan con las tradicionales herramientas que prolongaban la mano. Esta situación provoca experiencias aumentadas y una diversa alfabetización de los grupos que ingresan a la Universidad.

El propósito no es juzgar las competencias cognitivas de la nueva generación, sino interpretarlas. "Cualquiera que pretenda comunicarse con la generación videolectrónica debe tener en cuenta cómo funciona el cerebro colectivo post alfabético. La mente trata enseguida de desplazarse, de hallar otro objeto. La transferencia rápida procede por asociación y sustituye a la discriminación crítica" (Berardi, 2007).

Sujetos kinestésicos que aprenden mediante la praxis, perciben la información de manera abstracta, y construyen conocimiento aplicando teorías e hipótesis desde el sentido común. McLuhan sostuvo que cuando a la tecnología alfabética le sucede la electrónica y, en consecuencia, a lo secuencial le sucede lo simultáneo, la comunicación discursiva abre paso a formas de comunicación no discursivas, y el pensamiento mítico tiende a prevalecer sobre el pensamiento lógico-crítico.

"El proceso de trabajo es movilización constante de la atención y las semio-mercancías que constituyen el objeto principal del consumo contemporáneo demandan tiempo mental, atención y movilización ininterrumpida de las facultades cognitivas.” (Berardi, 2007)
El estado de situación esbozado implica reconocer un nuevo "paisaje textual" (Lankshear \& Snyder, 2000) y perceptual que requiere ser atendido a través de estrategias que atiendan los diferentes niveles de alfabetización: lectura, escritura y alfabetización digital.

La alfabetización académica, definida en términos de Carlino como la adquisición de un complejo conjunto de nociones y estrategias necesarias "para participar de la cultura discursiva de las disciplinas", implica pensar que leer, escribir y proyectar son sinónimos de aprendizaje. En definitiva, las actividades de lectura y escritura académica son actividades esenciales, pues participar de un campo del saber implica conocer el discurso de la disciplina y ser capaz de producirlo.

Intentar un polialfabetismo académico implica entonces reconocer que existen diferentes tipos de lenguajes que portan signos y sintaxis específicas, y que en la actualidad el lenguaje multimodal se torna un requerimiento de época que aumenta las diferencias, introduciendo una nueva problemática en la educación, que a la vez se presenta como posibilidad de experimentación de estrategias didácticas en relación a un alumno ingresante de pensamiento fragmentario vinculado más a operaciones kinestésicas que a operaciones lógico-formales.

Esta problemática socio-técnica, que está interdefinida por las dimensiones epistemológica, estética, ética y tecnológica, supone la organización de estrategias complejas de incorporación efectiva de la potencialidad de las TIC a la educación presencial, integrando miradas, valoraciones y aportes diversos (Mines, Tosello, 2011) para promover el diálogo multidireccional entre sujetos, conocimientos, espacios y medios (Bessone, 2007). 
Es preciso pues, delinear un proyecto educativo que acompañe un cambio epistemológico y metodológico por parte de los educadores, en el cual el rol docente se traslade de transmitir información, a guiar las búsquedas de los alumnos, ayudándoles a plantearse las preguntas adecuadas, despertándoles la curiosidad, acompanándolos para acceder a la información que necesitan y aprender a trabajar en equipo, competencias necesarias para el mundo del trabajo. Expresado en términos de Carlino: "sustitución del paradigma de la transmisión al paradigma de la transacción”, donde el conocimiento situado y la polialfabetización académica pueden resultar cambios posibles.

En la línea de pensamiento esbozado, toma de valor el concepto de "conocimiento situado". Donna Haraway, desde una perspectiva de género, reconoce la necesidad de que, independientemente del tipo de método empleado, la enseñanza parta de la subjetividad y el contexto cultural, así como de un punto de vista explícito y argumentado. Por eso el conocimiento siempre será parcial y situado, reconociendo además que de la integración de todas las visiones es posible un mejor acercamiento a la realidad. Es por ello que trata de asumir la parcialidad, a la vez que la validez de ese conocimiento es real, desde el punto de vista de quien lo genera.

Tal reconocimiento requiere desarrollar propuestas flexibles y de alta calidad, avanzando hacia un sistema que integre los estudios presenciales con los de educación a distancia, promoviendo una mayor incorporación de las TIC en aquellos. Estos desarrollos se constituirán en propuestas centrales para favorecer un acceso creciente y sostenido a una educación inclusiva (UNL, 2010).

En relación al estado de situación planteado y en el caso particular de la arquitectura, la ampliación y diversidad del discurso disciplinar y una alfabetización digital heterogénea, producen rupturas en los tradicionales modos de entender y abordar los procesos educativos, y presentan la posibilidad de experimentar didácticas que promuevan lógicas múltiples y colaborativas para pensar, diseñar y materializar las ideas.

Lógicas de ideación, fabricación y comunicación asociadas al lenguaje de los nuevos medios, y lógicas de tectonicidad vinculadas a poéticas de expresión material, se contrastan y complementan como un requerimiento de época; situaciones que requieren ser atendidas especialmente en la currícula y que requieren repensar qué y cómo enseñar.

\section{Metodología y materiales}

Desde el enfoque que plantea el marco de la Investigación-Acción se realizan diferentes indagaciones en las que participan estudiantes y docentes del primer año de Arquitectura, quienes como comunidad de aprendizaje y en espacios educativos híbridos, desarrollan actividades en dos entornos de aprendizaje: el Taller Curricular y Talleres Experimentales.

La investigación presenta dos momentos de indagación extracurricular y una posterior aplicación al Taller de Proyecto Arquitectónico 1.

- Primer momento: formulación de nociones y operaciones básicas presentadas como herramientas conceptuales y operativas en relación al objeto de estudio y al contexto.
Reconocimiento de la diversidad de alfabetización y oferta de un sistema análogo y otro paramétrico para la generación formal.

- Segundo momento: desde los resultados obtenidos se propone un trabajo integrado entre alumnos y auxiliares docentes de las asignaturas: Taller de Proyecto I, Introducción a la Tecnología, Matemática y Morfología, con el propósito de investigar el vínculo entre la generación de la forma (estructural y de envolventes), materiales y contexto.

- Tercer momento: incorporación al grado como oferta a los alumnos, en relación al tipo y nivel de alfabetización.

El registro y la clasificación de los datos se sustentan en la observación del diálogo multidireccional entre sujetos, objeto de conocimiento, espacios y medios. El entorno para el análisis y la interpretación de cada experiencia controlada, son los seminarios-taller abordados desde diversos campos como ejercicio de interpretación interactiva.

\section{Taller Experimental de Indagaciones Formales}

Definición del problema de diseño: "Resolución de unidades simples, plausibles de ser repetibles en el ambiente público".

\section{Objetivo general:}

Realizar un ensayo proyectual a partir del principio de "transformación”. Del concepto de línea y/o plano, a la expresión material de los sistemas de soporte y soportados.

Desde tales consignas formuladas para el Taller Curricular, se aborda la experiencia desde el paradigma educativo de la "transacción" (Carlino, 2005) y se incorpora a la currícula tradicional del Taller de Proyecto 1 el concepto de lógica proyectual presentando tres tipos de lógicas: del concepto, del material y paramétricas.

\section{Objetivos particulares:}

- Ensayar lógicas paramétricas + fabricación digital como herramientas de diseño.

- Evaluar potencialidades y riesgos de su aplicación en los talleres iniciales.

- Realizar una primera comparación entre procesos y resultados obtenidos en los dos espacios de enseńanza.

\section{Desarrollo}

En relación al cambio de paradigma planteado, la propuesta didáctica intenta cambiar el eje del discurso pedagógico y centrar sus estrategias en la oferta de herramientas conceptuales y operacionales que se traman con los sentidos, deseos y conocimientos previos del alumno (mundo referencial). A través de los principios de Introducción, Reintroducción, Selección, Economía de medios y tiempos, se pretende trabajar en el intersticio de la relación que el alumno establece con el conocimiento, mediante la interacción de los espacios físico y virtual, ensayando estrategias de indagación y trabajo colaborativo.

\section{Introducción:}

Las herramientas conceptuales se plantean desde tres abordajes: simbólico, geométrico y fenoménico. Como primer nivel 

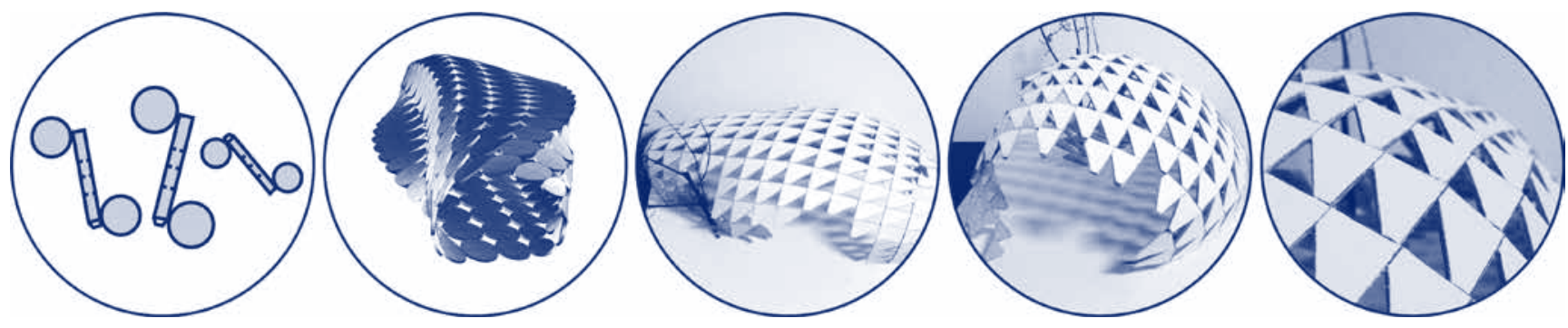

Figura 1: Proceso de ideación incorporando componentes. Maqueta digital y análoga (cartón).

de aproximación se pretende que el ingresante inicie el proceso de aprendizaje proyectual centrando los ejercicios en tres lógicas factibles de articular entre sí: lógica conceptual; lógica de generación-producción (geométrica); y lógica estructural-material (técnica y expresiva).

Desde la lógica conceptual se pretenden vincular sujeto, objeto, uso y contexto (ambiente) como primera aproximación a la dimensión simbólica.

Desde el abordaje de la lógica de generación y producción se presentan los elementos primarios de la forma y dispositivos de generación de la dimensión euclidiana, presentando como otras dimensiones posibles los sistemas de diseño paramétrico. Articulaciones y encuentros de líneas y planos definen la estructura y las envolventes.

Una lógica estructural-material se asocia a los elementos o componentes de generación primaria, reconociendo elementos de soporte, cerramientos y mixtos tanto en sus aspectos técnicos como expresivos, en relación a los elementos: agua, tierra, aire y fuego.

\section{Reintroducción.}

Se retrabajan conceptos referidos a tecnología y ambiente como variables de abordaje y se asocian éstos con la posibilidad de representarlos a partir de parámetros (forma, usos y tecnologías en relación al ambiente), reintroduciendo aspectos estudiados en las asignaturas Matemática, Sistemas de Representación, Morfología e Introducción a la Tecnología.

\section{Selección:}

Se propone al estudiante, de acuerdo a su nivel de alfabetización digital y su interés en participar, un cursado paralelo al taller curricular en el que se lo introduce en operaciones paramétricas básicas y potencialidades que el medio digital presenta para transformar en dato proyectual los análisis realizados e iniciar el desarrollo de un pensamiento proyectual ampliado.

\section{Economía de medios y tiempos:}

Una vez presentadas las herramientas para operar en ambos campos, se ofrece al alumno la posibilidad de trabajar con el medio que desee para el proceso de ideación, generando instancias de asociación y trabajo colaborativo. Para esta experiencia en particular, se ofrecen como instrumentos para la generación de la forma y su posterior fabricación:

Elementos geométricos primarios: línea y plano. Principios Ordenadores: transformación, repetición, cambio escalar, autosimilitud, etc. Componentes, familias y operaciones de diseño paramétrico: planos calados, secciones y costillas. Dispositivos de sintaxis: auto similitud, cambio escalar, convergencia, continuidad, conectividad, etc. Para la materialización se propone: madera, metal, vidrio y tela.

\section{El objeto de diseño}

La consigna es diseñar y fabricar un objeto de uso urbano desmontable, repetible y transportable, vinculado a la escala humana y al lugar.

Herramientas operacionales: software Rhinoceros y Grasshopper. Materiales: madera y metal. Dos entornos de aprendizaje: presencial y a distancia.

Tiempos: 4 semanas. Dos horas de clases presenciales + interacciones en grupo cerrado en Facebook, acorde a la demanda de los alumnos.

\section{Resultados}

Desde la sistematización del registro de observación se ha detectado el diálogo multidireccional entre sujetos, competencias, espacios y medios. La experiencia posibilita verificar cambios en la tradicional triada didáctica: docente-alumno-conocimiento. El docente se posiciona como mediador entre el software utilizado y los alumnos. La clase se transforma en una comunidad de investigación en la que los diferentes miembros utilizan un lenguaje común, que corresponde con el "paradigma de la transacción". Los alumnos asisten con gran interés y colaboran entre ellos. Se visualizan diferentes niveles de aprendizaje en relación al nivel de alfabetización digital inicial.

Desde la sistematización de la encuesta dirigida a los estudiantes, se reconoce la comprensión del diseño paramétrico como un proceso complementario que se constituye a su vez en instrumento que potencia la vinculación de contenidos específicos de Matemática y Sistemas de Representación, asignaturas pertenecientes al Ciclo Básico de la carrera, otorgando sentido a las articulaciones deseadas entre criterios formales, estructurales, materiales y ambientales; presentándose además el modelo tridimensional como posibilidad de una mayor comprensión espacial de los elementos que componen la forma.

Si bien es un ensayo inicial, el presente trabajo pretende constituir un aporte a la discusión respecto de la necesidad de actualización de la didáctica proyectual en los talleres iniciales de la carrera de Arquitectura, a través de sus autores y proyectos.

\section{Caso 1- Gómez: Componente (Fig. 1)}

"Diseñar con palabras no ver los objetos, jugar... ponía una cosa salía otra, investigar, experimentar, incentivo... es otra lógica que no excluye el croquis, la maqueta, vas probando, lo hacía en mis ratos libres"... "tener el vector asignado posibilita controlar a partir del calado de pieles".

\section{Caso 2- Thiel: Costillas (Fig. 2)}

"Potencialidad para pensar, despertar la curiosidad, no tuve dificultades con lo paramétrico, si con los componentes en el proceso de fabricación 
digital, es lento, no asi el de costillas o haciendo una especie de piel calada."...ciertas aplicaciones... vector solar... visuales... ocultar... eficiencia, pero encuentro contradicciones con las formas que se generan, no las percibo como eficientes".

Caso 3-Planells: Cortes Planares (Fig. ᄏ)

"Como sos ingresante te abre la cabeza para pensar más allá de 4 paredes y un techo, pensar en otro tipo de formas. Seguís buscando... Es complementario, siempre necesitas unos croquis..."

"Encuentro articulaciones con lo enseñado en tecnología en relación a tensiones, materiales y acondicionamiento ambiental."

Caso 4- Giovannini: Despliegue / Pliegue (Fig. 4)

"Diseñar más libre, curvas a partir de un sentido y motivo, me costó entenderlo, muy buenas las clases de Matías, investigar, experimentar, incentivo, me llevó tiempo pero ahora lo apliqué en el Taller. Lo voy a seguir investigando en las vacaciones."

"Permite una mejor interpretación y articulación del reconocimiento de fuerzas e interpretación y respuestas al calor y las orientaciones".

\section{Conclusiones}

Desde el reconocimiento de estos resultados provisorios, la experiencia abre la posibilidad de pensar en una propuesta didáctica que articule

Figura 2: Proceso de ideación utilizando lógicas de costillas estructurales/ cerramiento. Maqueta digital y análoga (mdf).

Figura 3: Proceso de ideación a través de cortes planares. Maqueta digital y análoga (mdf).

Figura 4: Proceso de ideación inmerso en estrategias de despliegue/pliegue. Maqueta digital y análoga (papel). diferentes niveles de alfabetización, a través de estrategias de colaboración y una docencia articulada atenta a un aprendizaje situado.

Las nuevas estrategias proyectuales, como las de diseño paramétrico y fabricación digital, deben ser enmarcadas en una estructura integral: propuestas didácticas innovadoras asociadas a un nuevo paradigma educativo, interdefinido por las dimensiones epistemológica, tecnológica, estética y ética.

\section{Agradecimientos}

Este trabajo es parte del proyecto de investigación CAI+D 2011 N³56 "Diseño, desarrollo y evaluación de espacios-interfaz destinados a actividades de docencia, investigación y extensión en la universidad pública argentina”, subsidiado por la Universidad Nacional del Litoral, Santa Fe, Argentina.

\section{Referencias}

Lankshear \& Snyder. (2000) Teachers and Technoliteracy: Managing Literacy, Technology and Learning in Schools. Allen \& Unwin. St. Leonards, NSW, Australie.

Mines, P., Tosello, M. (2011). Nuevos Escenarios Educativos. En VIII Congreso Nacional de Sociedad Estudios Morfológicos de Argentina, SEMA. 82-85. Santa Fe: UNL.

Berardi, F. (2007), Generación post-alfa. Patologías e imaginarios en el semiocapitalismo, Bs. As., Tinta Limón, 7-31.

Bessone, M. (2007). Tesis de Maestría: Consecuencias de la Aplicación de los medios digitales en la enseñanza de la Arquitectura.

Carlino, P.(2005). Escribir, leer y aprender en la Universidad. Editorial FCE. Buenos Aires. Argentina

Universidad Nacional del Litoral (2010). Plan de Desarrollo Institucional 2010-2019. Alta calidad en enseñanza, investigación y extensión del conocimiento (LOP II).
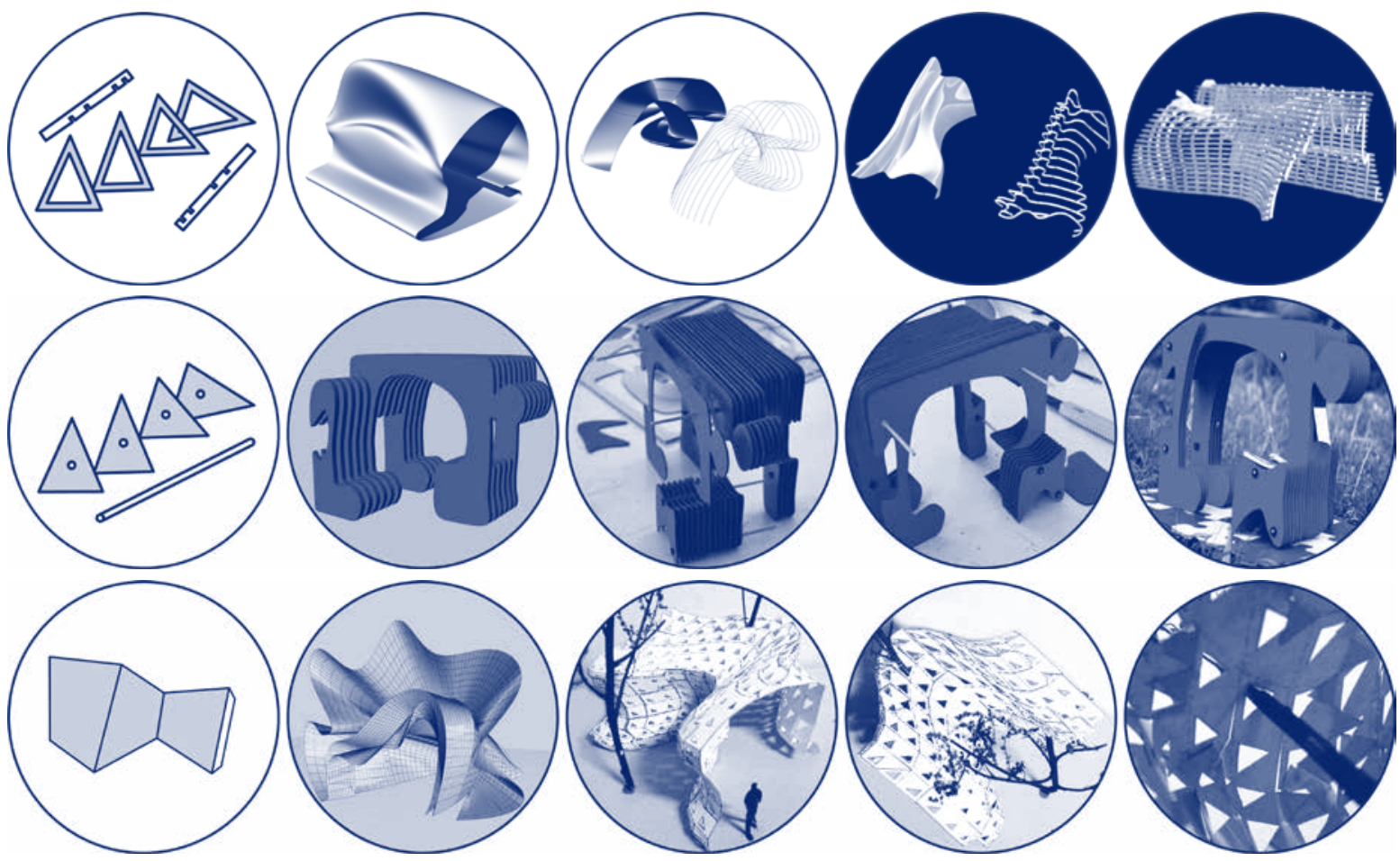